\title{
TRANSIT TIMING OBSERVATIONS FROM KEPLER. VI. POTENTIALLY INTERESTING CANDIDATE SYSTEMS FROM FOURIER-BASED STATISTICAL TESTS
}

\author{
Jason H. StefFen ${ }^{1}$, Eric B. Ford ${ }^{2}$, Jason F. Rowe ${ }^{3,4}$, Daniel C. Fabrycky ${ }^{5,12}$, Matthew J. Holman ${ }^{6}$, William F. Welsh ${ }^{7}$, \\ Natalie M. Batalha ${ }^{8}$, William J. Borucki ${ }^{3}$, Steve Bryson ${ }^{3}$, Douglas A. Caldwell ${ }^{3,4}$, David R. Ciardi ${ }^{9}$, \\ Jon M. Jenkins ${ }^{3,4}$, Hans KJeldsen ${ }^{10}$, David G. Koch ${ }^{3}$, Andrej PrŠa ${ }^{11}$, Dwight T. SANDerfer ${ }^{3}$, \\ Shawn SeAder ${ }^{3}$, AND Joseph D. TWiCKEN ${ }^{3,4}$ \\ ${ }^{1}$ Fermilab Center for Particle Astrophysics, P.O. Box 500, MS 127, Batavia, IL 60510, USA; jsteffen@ @nal. gov \\ 2 Astronomy Department, University of Florida, 211 Bryant Space Sciences Center, Gainesville, FL 32111, USA \\ ${ }^{3}$ NASA Ames Research Center, Moffett Field, CA 94035, USA \\ ${ }^{4}$ SETI Institute, Mountain View, CA 94043, USA \\ ${ }^{5}$ UCO/Lick Observatory, University of California, Santa Cruz, CA 95064, USA \\ ${ }^{6}$ Harvard-Smithsonian Center for Astrophysics, 60 Garden Street, Cambridge, MA 02138, USA \\ ${ }^{7}$ Astronomy Department, San Diego State University, San Diego, CA 92182-1221, USA \\ ${ }^{8}$ Department of Physics and Astronomy, San Jose State University, San Jose, CA 95192, USA \\ ${ }^{9}$ NASA Exoplanet Science Institute/California Institute of Technology, Pasadena, CA 91125, USA \\ ${ }^{10}$ Department of Physics and Astronomy, Aarhus University, DK-8000 Aarhus C, Denmark \\ ${ }^{11}$ Department of Astronomy and Astrophysics, Villanova University, 800 East Lancaster Avenue, Villanova, PA 19085, USA \\ Received 2012 January 9; accepted 2012 June 20; published 2012 August 27
}

\begin{abstract}
We analyze the deviations of transit times from a linear ephemeris for the Kepler Objects of Interest (KOI) through quarter six of science data. We conduct two statistical tests for all KOIs and a related statistical test for all pairs of KOIs in multi-transiting systems. These tests identify several systems which show potentially interesting transit timing variations (TTVs). Strong TTV systems have been valuable for the confirmation of planets and their mass measurements. Many of the systems identified in this study should prove fruitful for detailed TTV studies.
\end{abstract}

Key words: planetary systems - planets and satellites: detection - planets and satellites: dynamical evolution and stability - techniques: miscellaneous

Online-only material: color figures, machine-readable table

\section{INTRODUCTION}

In transiting exoplanet systems, the deviations from a constant orbital period caused by planet-planet dynamical interactions have proven to be very useful in detecting and characterizing the constituent planets. Transit timing variations (TTVs) for short timescale and resonant interactions are particularly useful (Agol et al. 2005; Holman \& Murray 2005) though secularly induced TTVs have also been studied (Miralda-Escudé 2002; Heyl \& Gladman 2007). The Kepler mission has profited greatly from the use of TTVs, having used them extensively in the confirmation of a significant fraction of its currently confirmed planets: Kepler-9 (Holman et al. 2010), Kepler-11 (Lissauer et al. 2011), Kepler-18 (Cochran et al. 2011), and Kepler systems 23-34 (Ford et al. 2012a; Fabrycky et al. 2012; Steffen et al. 2012a). TTVs have given: important mass measurements for smaller planets that would be exceedingly difficult to obtain through radial velocity measurements; stringent limits on the presence of small planets near mean-motion resonance (MMR) with hot Jupiters (Steffen \& Agol 2005; Steffen et al. 2012b) with consequent constraints on planet formation and dynamical evolution; and the identification of non-transiting planets such as Kepler-19 (Ballard et al. 2011) —with as-yet undetermined orbital properties.

With the initial release of two quarters of data in 2011 (Borucki et al. 2011) there was an accompanying paper discussing potential TTV candidates (Ford et al. 2011). Now, with

\footnotetext{
${ }^{12}$ Hubble Fellow.
}

the release of more than a year of additional data, we revisit the task of identifying systems that show interesting TTV signatures here and in a companion paper (Ford et al. 2012b) using the transit times reported in Rowe et al. (2012). A number of these systems have already been identified, studied, and announced. However, several new systems have been the subject of only cursory investigation and merit further scrutiny. The increased time baseline of the Kepler data through quarter six (Q6) provides many more systems with interesting TTV features as typical timescales for large TTV signals are tens of orbits-which are only now available. With continued operations the scientific yield from Kepler and from TTVs specifically will be particularly valuable to the study of planets as many of the orbital and physical properties of planets with small sizes and masses can only be gleaned through TTV analysis. The extension of the Kepler mission, with its increased time baseline for TTV studies, will present an opportunity to learn about planetsnotably including terrestrial planets and planets near the habitable zone- that will not be rivaled for many decades.

In what follows, we present the results of a few straightforward statistical tests on the transit times of known Kepler Objects of Interest (KOI). Two of these tests are applied to all individual KOIs, while a third test is applied to pairs of KOIs in the same system in an effort to use correlated TTV signals as a means to improve sensitivity to dynamical interactions. The point of these tests is not to assign rigorous absolute probabilities for the detection of a TTV signal; rather, it is to identify systems that merit additional scrutiny. The results of the tests are presented in a single, large table for all analyzed systems (Table 1). 
Table 1

Period, Number of Transits, the Results of the S/N of the Best-fit Sinusoidal Amplitude, the $p$-value for the $F$-ratio Test, and $\Xi_{\max }$ for Each KOI

\begin{tabular}{llllll}
\hline \hline KOI & $\begin{array}{l}\text { Period } \\
\text { (days) }\end{array}$ & $N_{t}$ & $\mathrm{~S} / \mathrm{N}$ & $p$-value & $\Xi_{\max }{ }^{\mathrm{a}}$ \\
\hline
\end{tabular}

\begin{tabular}{lrrrrr}
$\vdots$ & & & & & \\
64.01 & 1.951 & 215 & 5.474 & 0.4106 & \\
69.01 & 4.727 & 98 & 4.854 & 0.3954 & \\
70.01 & 10.854 & 40 & 3.731 & 0.3782 & 4.685 \\
70.02 & 3.696 & 120 & 6.514 & 0.3831 & 5.301 \\
70.05 & 19.578 & 23 & 5.163 & 0.2895 & \\
72.01 & 0.837 & 432 & 5.413 & 0.4211 & 0.676 \\
72.02 & 45.294 & 10 & 4.663 & 0.1601 & \\
$\vdots$ & & & & & \\
\hline
\end{tabular}

Note. ${ }^{a} \Xi_{\max }$ values are given for each KOI with its nearest exterior neighbor that satisfies the selection criteria for analysis (i.e., its exterior neighbor that is also reported in this table)

(This table is available in its entirety in a machine-readable form in the online journal. A portion is shown here for guidance regarding its form and content.)

\section{DATA REDUCTION}

We use KOIs from the planet candidate table reported in Borucki et al. (2011) and Batalha et al. (2012). The transit times for each KOI are determined following the method described in Ford et al. (2011) and are given in tabular form in Rowe et al. (2012). Frequently with that method there are some transit times that have anomalously large deviations from a linear ephemeris or that have unusually large error bars. In most cases, these anomalies are due to some source of photometric noise or transit detections with low significance rather than from planetary dynamics. However, in cases in which TTVs are larger than the transit duration, the algorithm may fail to find the correct solution, with the optimization algorithm not reaching the global minimum even if the individual transits are highly significant. In those cases, we rerun the algorithm with the initial guesses close enough to the true transit times so that the algorithm can model them correctly.

In order to remove non-planetary anomalous TTVs from our analysis, we reduce our timing data by requiring that the mean signal-to-noise ratio $(\mathrm{S} / \mathrm{N})$ per transit for a given KOI exceed 3. We eliminate all transit times where the timing residuals deviate from a linear ephemeris by more than four times the median absolute deviation of all timing residuals. We also eliminate all transit times where the timing uncertainty is larger than twice the median error of all timing uncertainties for that system. Once this data reduction criterion has been applied, we fit a new linear ephemeris and conduct our remaining analysis on the resulting timing residuals. Table 1 shows the KOIs studied here (1481 with 1009 that satisfy the selection criteria) along with the results of the statistical tests outlined below.

\section{INDIVIDUAL OBJECTS}

The first analysis we present is applied to each individual KOI. Here, we look for a sinusoidal TTV signal with an arbitrary amplitude, phase, and period. TTV signals for systems in or near MMR are expected to be roughly sinusoidal as the description of their motion is dominated by a single sinusoidal component that corresponds to that MMR (Agol et al. 2005). While systems farther from MMR have multi-sinusoidal TTV signatures, the amplitude of the signal is significantly weaker and would not

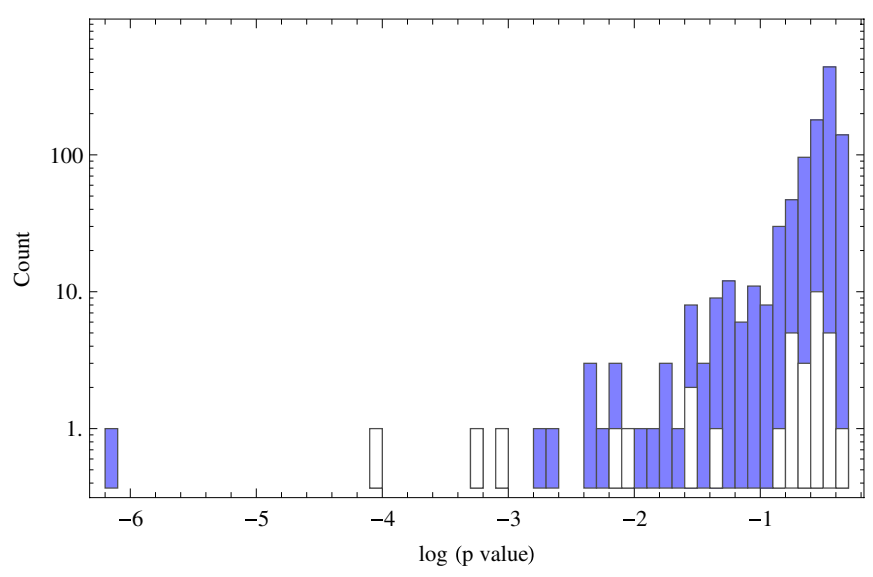

Figure 1. Histogram of the $p$-values resulting from the $F$-ratio test. The $p$-values from known multiplanet systems are the light-colored bars. The two most significant systems with this test are KOI-227 and Kepler-9.

(A color version of this figure is available in the online journal.)

likely be marked as significant in this study (Agol et al. 2005; Steffen 2006). We compare this single-sinusoid model to the null model where the transit times are fit to a linear ephemeris using the standard $F$-ratio test with two and five parameters for the null model and the sinusoidal model, respectively. This method was first applied to a sample of hot Jupiter candidates in Steffen et al. (2012b).

We do not claim that the results of this test give an accurate estimate of the statistical significance of a TTV signal, rather that this test can identify interesting systems that merit further scrutiny-as is the goal of this paper. The results of the $F$-ratio test ( $p$-values that represent the probability that the twoparameter, null model is the correct model of the two models being compared) are shown in Figure 1. We note that the KOIs in light colors in this and in all figures are for known planets or well-studied KOIs and include: 84 (Kepler-19), 137 (Kepler18), 157 (Kepler-11), 168 (Kepler-23), 244 (Kepler-25), 250 (Kepler-26), 377 (Kepler-9), 738 (Kepler-29), 806 (Kepler-30), 841 (Kepler-27), 870 (Kepler-28), 935 (Kepler-31), 952 (Kepler32), and 1102 (Kepler-24).

There are a few planet candidates among the group of wellstudied objects that have relatively small $p$-values, notably KOI-806 (Kepler-30) (Tingley et al. 2011; Fabrycky et al. 2012) and KOI-377 (Kepler-9) (Holman et al. 2010). However, it is noteworthy that most of the multi-transiting systems that were studied for anticorrelated TTV signatures in Ford et al. (2012a), Fabrycky et al. (2012), and Steffen et al. (2012a) and the planets in Kepler-11 (Lissauer et al. 2011) do not have particularly low $p$-values. This is further indication of the importance of multi-transiting planetary systems for the purposes of planet confirmation and dynamical interpretation. A correlation analysis, as is done in Ford et al. (2012a), Fabrycky et al. (2012), and Steffen et al. (2012a), and in the next section of this work, can identify otherwise weak individual candidates. For the $F$-ratio test, systems that have $p$-values below 0.02 and that have 10 or more transits are likely to be the most interesting and include (in order of significance) KOIs: 227, 377 (Kepler-9), 806 (Kepler-30), 142, 277 (Kepler-36; Carter et al. 2012), 1573, 448, and 918 - while still others have undoubtedly interesting signals.

A second analysis for the individual KOIs was to record the ratio of the amplitude of the best-fitting sinusoidal model to the uncertainty in that amplitude (the $\mathrm{S} / \mathrm{N}$ of the sinusoid 


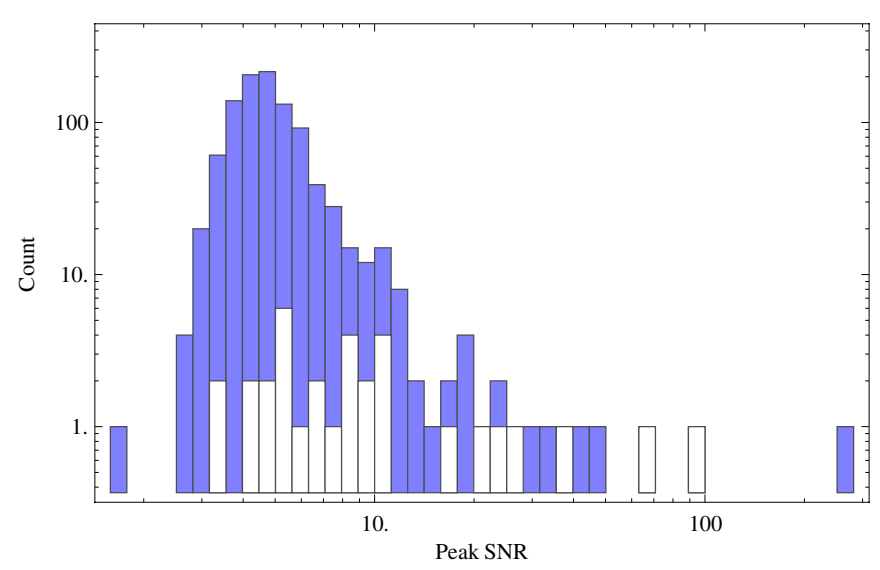

Figure 2. Histogram of the $\mathrm{S} / \mathrm{N}$ values resulting from the $\mathrm{S} / \mathrm{N}$ test. Known multibody systems are the light-colored bars. Again, the two most significant systems identified by this test are KOI-227 and Kepler-9. However, KOI-142 and KOI-1573 also have very large $\mathrm{S} / \mathrm{N}$ detections.

(A color version of this figure is available in the online journal.)

amplitude fit). This $\mathrm{S} / \mathrm{N}$ statistic is given by

$$
\mathrm{S} / \mathrm{N}=\left(\left(A^{2}+B^{2}\right)\left(\sigma_{A}^{-2}+\sigma_{B}^{-2}\right)\right)^{1 / 2},
$$

where $A$ and $B$ are the amplitudes of the best-fitting sine and cosine components and $\sigma_{A}$ and $\sigma_{B}$ are the uncertainties in those amplitudes (see Equation (2) below).

Figure 2 shows the results of this test for all KOIs. As with the $F$-ratio test, the $\mathrm{S} / \mathrm{N}$ test shows several systems with sinusoidal amplitudes detected with a large $\mathrm{S} / \mathrm{N}$-including many that have not been investigated in depth. The top candidates from this test with an $\mathrm{S} / \mathrm{N}$ greater than 20 and more than 10 transits include (in order of significance) KOIs: 227, 377 (Kepler9), 142, 1573, 806, 984, 13, 277 (Kepler-36), 473, and 137 (Kepler-18). It is noteworthy that not all of the top candidates from the $F$-ratio test are top candidates for the $\mathrm{S} / \mathrm{N}$ test and vice versa. We also point out that in some cases, the tests identify some clearly bad candidate systems. For example, the $\mathrm{S} / \mathrm{N}$ test identifies KOI-13 with high significance-while its timing residuals are caused by an interesting mix of stellar variability and planet-star dynamics, but not an additional planet (Szabó et al. 2012). Figure 3 shows the TTV signal for the three most significant candidates for both the $\mathrm{S} / \mathrm{N}$ test and the $F$-ratio test provided they have more than 10 transit times (KOIs 227, 142, and 1573). None of these have yet been claimed as confirmed planets.

\section{OBJECT PAIRS}

For pairs of planets, an additional diagnostic is to look for anticorrelation in the TTV signal. As planets interact with each other, we expect many of their TTV signatures to be anticorrelated due to basic effects from conservation of energy and angular momentum-especially when a system is near MMR. It is possible to have short-timescale-correlated TTV signals in systems farther from resonance or when there is significant precession in one of the objects (Steffen et al. 2012a; Fabrycky et al. 2012; Ford et al. 2012a). Using an anticorrelation measurement can give much more power to distinguish real TTV signals from noise, as one would expect timing noise to be positively correlated at best and uncorrelated at worst for minimally interacting planets (some dynamical scenarios can produce positively correlated residuals on short timescales).
Following the procedure outlined in Steffen et al. (2012a), we calculate $\Xi_{\max }$ for each pair of KOIs that satisfy the selection criteria outlined above for all of the systems with multiple transiting objects. This results in 155 systems with 262 total pairs that meet the selection criteria. The statistic $\Xi_{\max }$ is found from a periodogram generated by fitting the TTV signal to a set of sinusoidal functions with specific periods:

$$
f_{i}=A \sin \left(\frac{2 \pi t}{P_{i}}\right)+B \cos \left(\frac{2 \pi t}{P_{i}}\right)+C,
$$

where $A, B$, and $C$ are model parameters and $P_{i}$ is the test timescale.

The fitted values for $A$ and $B$ and their measured uncertainties, $\sigma_{A}$ and $\sigma_{B}$, are then used to calculate $\Xi$ for each of the sampled periods using

$$
\Xi=-\left(\frac{A_{1} A_{2}}{\sigma_{A 1} \sigma_{A 2}}+\frac{B_{1} B_{2}}{\sigma_{B 1} \sigma_{B 2}}\right),
$$

where the " 1 " and " 2 " subscripts correspond to the two objects. Finally, the maximum value that $\Xi$ has for a given candidate pair, $\Xi_{\max }$, is the statistic we choose to determine the significance of any interaction between the two objects. The square root of $\Xi_{\max }$ is approximately the significance in terms of $\sigma$ for Gaussian deviates, though we note that an accurate determination of the significance is best done via a Monte Carlo simulation (see Ford et al. 2012a; Fabrycky et al. 2012; Steffen et al. 2012a).

Figure 4 shows the results of this analysis for all KOI pairs. Many of the most significant pairs are in previously identified systems (denoted by the light bars) with Kepler-9 and Kepler-18 being the most significant detections. Still, several pairs have not been previously identified in the literature including KOI-904. Other significant pairs may be found by including systems with fewer transits (e.g., Kepler-30) or with transits detected with less significance, however, expanding the analysis down to such systems results in many more spurious detections. The example of KOI-904 shows how correlation statistics have more power to identify potential TTV systems than simply tests on individual KOIs - the KOI-904 system ranks highly with the correlation test, but the individual objects barely make the top 100 for the $F$-ratio test and are roughly 50th and 100 th for the $\mathrm{S} / \mathrm{N}$ test. Table 1 includes a subset of all calculated $\Xi_{\max }$ values-specifically, the $\Xi_{\max }$ value found for a given candidate and its neighbor with the next largest orbital period that satisfies the sample selection criteria.

The companion paper by Ford et al. (2012b) has similar tests using simple parametric modeling such as quadratic or cubic fits to the TTV signals. Using those methods, several of the strongest systems do not appear strong with the Fourier methods and vice versa-and the relative ranking is quite different. This is to be expected as only in limiting cases do the two models have similar functional form. Moreover, trigonometric functions require a specific relationship between the polynomial powers while generic power-law models do not. Trigonometric functions have a strong physical motivation (as they are the natural basis to solve the equations of motions of gravitating systems) and they allow for more straightforward correlation tests at specific frequencies as is done in this section. Nevertheless, the power-law models of Ford et al. (2012b) can be a faster means of finding interesting systems - especially when there are fewer data to analyze. The full power of the Fourier methods is best realized when multiple cycles exist in the TTV residuals, which will be increasingly the case as more Kepler data become available. 

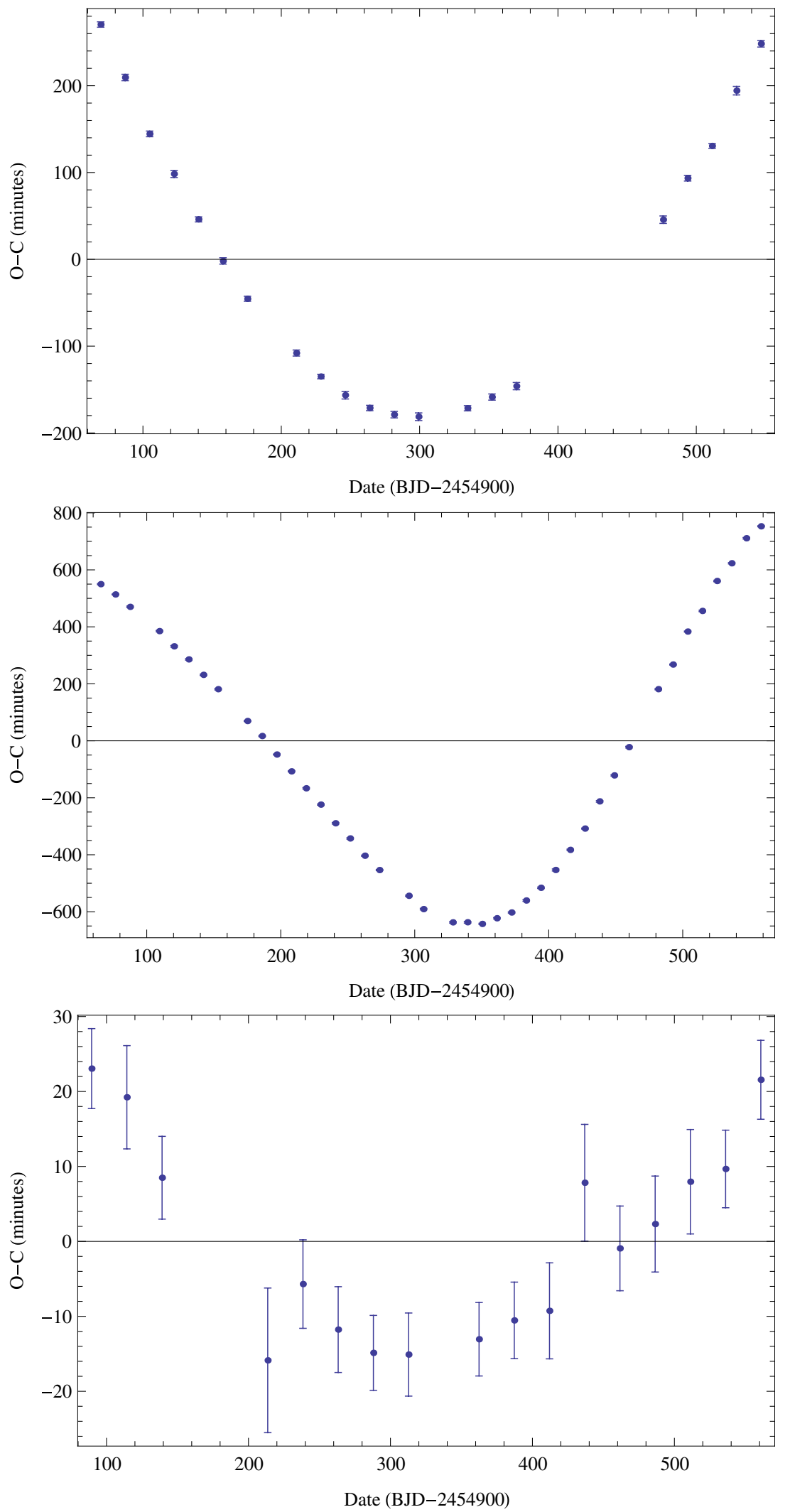

Figure 3. Residuals from a linear ephemeris (observed minus calculated) for the three planet candidates with the most significant results from the $\mathrm{S} / \mathrm{N}$ test and the $F$-ratio test that have not yet been claimed as planets and that have more than 10 measured transit times. KOI-227.01 is the top panel, KOI-142.01 is the middle panel, and KOI-1573.01 is the lower panel.

(A color version of this figure is available in the online journal.) 


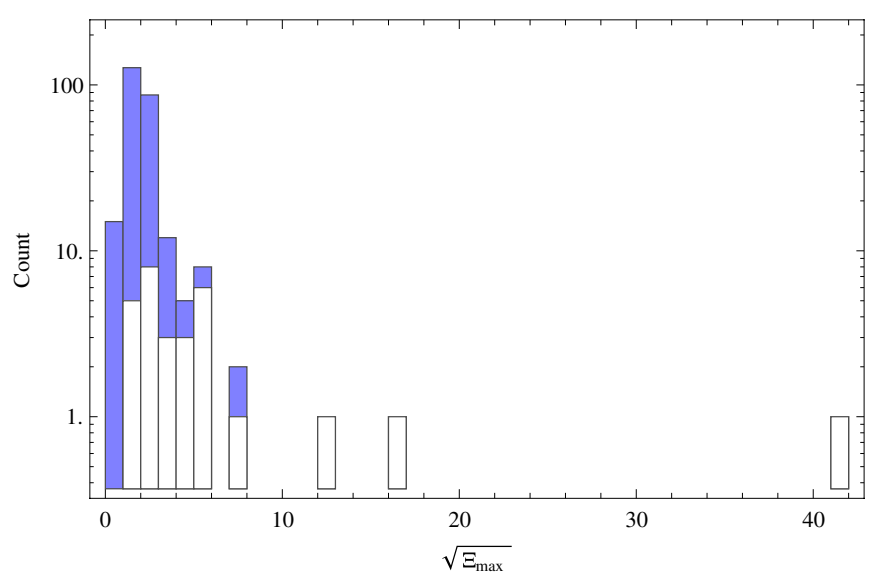

Figure 4. Histogram of $\sqrt{\Xi_{\max }}$ for all pairs of candidates. Pairs from wellstudied multiobject systems are shown with light-colored bars.

(A color version of this figure is available in the online journal.)

\section{CONCLUSIONS}

With the public release of Kepler data through Q6, many opportunities are available to study the detailed dynamics of Kepler candidate systems. We have conducted some straightforward statistical tests in an effort to identify systems that may be particularly fruitful. Some of the most interesting systems have been identified previously. However, many remain unexplored. Table 1 gives the results of each of these tests and can be used to identify systems that are worthy of additional scrutiny.

Kepler's unique data have enabled unprecedented scientific advances in the study of exoplanets and TTV analyses have played a central role in this endeavor. With these new data and the identification of additional TTV candidate systems, several important opportunities are enabled, such as the measurement of planetary masses, dynamical studies of MMR, the constraints on mutual inclinations, and the discovery of non-transiting exoplanets. These can, in turn, inform models of planet formation and dynamical evolution. Ultimately, TTV analysis is the only means currently available that can measure planet masses of terrestrial planets in the habitable zone of a Sun-like star. At present, its full potential has not been realized.

Funding for this mission is provided by NASA's Science Mission Directorate. J.H.S. acknowledges support by NASA under grant NNX08AR04G issued through the Kepler Participating Scientist Program. D.C.F. and J.A.C. acknowledge that support for this work was provided by NASA through Hubble Fellowship grants HF-51272.01-A and HF-51267.01-A awarded by the Space Telescope Science Institute, operated by the Association of Universities for Research in Astronomy, Inc., for NASA, under contract NAS 5-26555.

\section{REFERENCES}

Agol, E., Steffen, J., Sari, R., \& Clarkson, W. 2005, MNRAS, 359, 567 Ballard, S., Fabrycky, D., Fressin, F., et al. 2011, ApJ, 743, 200 Batalha, N. M., Rowe, J. F., Bryson, S. T., et al. 2012, ApJS (arXiv:1202.5852) Borucki, W. J., Koch, D. G., Basri, G., et al. 2011, ApJ, 736, 19 Carter, J. A., Agol, E., Chaplin, W. J., et al. 2012, Science, in press Cochran, W. D., Fabrycky, D. C., Torres, G., et al. 2011, ApJS, 197, 7 Fabrycky, D. C., Ford, E. B., Steffen, J.-H., et al. 2012, ApJ, 750, 114 Ford, E. B., Fabrycky, D. C., Steffen, J.-H., et al. 2012a, ApJ, 750, 113 Ford, E. B., Ragozzine, D., Rowe, J. F., et al. 2012b, ApJ, 756, 185 Ford, E. B., Rowe, J. F., Fabrycky, D. C., et al. 2011, ApJS, 197, 2 Heyl, J. S., \& Gladman, B. J. 2007, MNRAS, 377, 1511

Holman, M. J., Fabrycky, D. C., Ragozzine, D., et al. 2010, Science, 330, 51 Holman, M. J., \& Murray, N. W. 2005, Science, 307, 1288

Lissauer, J. J., Fabrycky, D. C., Ford, E. B., et al. 2011, Nature, 470, 53 Miralda-Escudé, J. 2002, ApJ, 564, 1019

Rowe, J. F., et al. 2012, ApJS, in preparation Steffen, J. H. 2006, PhD thesis, Univ. Washington Steffen, J. H., \& Agol, E. 2005, MNRAS, 364, L96

Steffen, J. H., Ford, E. B., Fabrycky, D. C., et al. 2012a, MNRAS, 421, 2342

Steffen, J. H., Ragozzine, D., Fabrycky, D. C., et al. 2012b, Proc. Natl. Acad. Sci., 109, 21, 7982

Szabó, G. M., Pál, A., Derekas, A., Simon, A. E., Szalai, T., \& Kiss, L. L. 2012, MNRAS, 421, L122

Tingley, B., Palle, E., Parviainen, H., et al. 2011, A\&A, 536, L9 